\title{
Transmission loss of thin membrane-type acoustic metamaterials with multi-masses
}

\author{
Guochang Lin ${ }^{\mathrm{a}}$, Songqiao Chen, Xuebo Yuan and Huifeng Tan \\ Center for Composite Material, Harbin Institute of Technology, Harbin 150001, China
}

\begin{abstract}
Membrane-type acoustic metamaterial can decrease sound transmission by $500 \%$ over mass law predictions in the frequency range of $100 \mathrm{~Hz}$ to $1000 \mathrm{~Hz}$. Membrane-type acoustic metamaterial stresses the structure design of elements, in order to reduce the best sound insulation frequency, broaden frequency range of the sound insulation and increase transmission loss(TL) magnitude. In this work, we established a membrane-type acoustic metamaterial element with high density polyethylene (HDPE) membrane and lead. Numerical simulation showed that this membrane-type acoustic metamaterial element has strong sound insulation effect at frequencies $(20 \mathrm{~Hz}-100 \mathrm{~Hz})$ and broadens the low frequency sound insulation range greatly. The central mass significantly influences the TL, and we found that the best sound insulation frequency can be acquired by adjusting the mass magnitude of centrally single mass. In addition, we designed a kind of membrane-type acoustic metamaterials with multimasses, and studied the sound insulation property by changing the number, distribution type and mass magnitude of surrounding masses on the membrane. Result showed that the number, distribution type and mass magnitude of surrounding masses have different impacts on the sound insulation frequency, the peak number of TL, and the frequency band of sound insulation
\end{abstract}

Keywords: membrane-type acoustic metamaterial; sound insulation; numerical simulation.

\section{Introduction}

In the engine of aerospace and aviation fields, sound insulation materials should have good acoustic insulation effect and light-weight structure. Membrane materials with advantage of light weight, are the focus of many researchers, which may be used in acoustic insulation application, especially in the aspects of low frequency noise. Membrane materials are regarded to break the restriction of the mass law. Takahashi[1] conducted theoretical study of sound absorption and sound transmission by developing a single permeable membrane. Yang and Mei[2] put forward a membrane-type metamaterial with negative effective mass density, which consisted of a circular elastic membrane, a mass attached in the center of the membrane and a rigid frame supporting the membrane. By a series of experiments and theoretical analysis, they discovered that the sound appears total reflection when the sound frequency range is $200 \mathrm{~Hz}-300 \mathrm{~Hz}$, and the mass density of entire structure is negative at the same time. The changes of mass magnitude and elastic modulus of the membrane influence the above frequency range, which can be used to acquire sound insulation effect at low frequencies. The

${ }^{a}$ Corresponding author : lingc@hit.edu.cn 
resonant frequency of the mass is single and narrow, we should design multi-layer membrane metamaterial structures if we hope to realize the noise reduction of broad frequency band. Yang[3] carried out experimental study in term of acoustic metamaterial in the frequency range of $50 \mathrm{~Hz}-$ $1000 \mathrm{~Hz}$, and the result showed that multi-layer structures improve the sound insulation effect.

Membrane-type acoustic metamaterial has become a new research pot because of the low frequency sound insulation, which can reduce the best sound insulation frequency, broaden the frequency band of sound insulation and increase the TL(Transmission Loss) magnitude by designing the structure element. Many researchers[4-11] studied the factors influence the sound insulation including membrane prestress, membrane thickness and material properties, and some mathematical analysis models were established accordingly. Their conclusions give some reference for the optimal structure design. Mei[12] fixed the rectangle membrane(with the thickness of $0.2 \mathrm{~mm}$ ) on the rigid frame, and several half circular plates are attached on the membrane, and this structure produces multi-resonances and absorbs the acoustic energy near resonant frequencies. Zhang[13] put forward a fast approach for calculating the sound transmission loss of the membrane-type acoustic metamaterial, and his result showed that the first trough and peak of the TL is related closely to the attached mass, and the second trough of the TL is dependent on the property of the membrane.In this work, we established a membrane-type locally resonant acoustic metamaterial model. Sound attenuation frequency range was reduced to $20 \mathrm{~Hz}-100 \mathrm{~Hz}$ by optimal design, and we also analyzed the influences of the number of small masses and the distribution type around the central mass on the sound insulation effect of this membrane-type metamaterial.

\section{Basic structure}

For designing the traditional sound insulation materials, the TL is calculated though the mass law[14] $\mathrm{TL}=-42+20 \lg f+20 \lg M(\mathrm{~dB})$ ( $f$ is the sound frequency, $M$ is the mass of the unit area of the sound insulation material. The TL of the traditional sound insulation materials is lower in the low frequency range $(50 \mathrm{~Hz}-200 \mathrm{~Hz})$ than that in the high frequency range(over $2000 \mathrm{~Hz})$. Based on the mass law, the calculated result shows that the TL increases by only $20 \mathrm{~dB}$ when the structure thickness extends tenfold. Therefore, in order to improve the sound insulation ability for the low frequency noise without markedly increased thickness, it is urgent to search for a special material breaking the restriction of the mass law. The acoustic metamaterial offers an appropriate solution for this.

Membrane-type acoustic metamaterial studied in this work consists of thin membrane, rigid mass and supporting frame. The Fig.1(a) presents a typical membrane-type acoustic metamaterial structure element, elastic membrane is fixed on the rigid square frame, and a rigid mass is adhered on the center of the membrane. The Fig.1(b) shows a kind of two dimensional membrane-type acoustic metamaterials, which is composed of $n \times n$ structure elements with periodic arrangement.

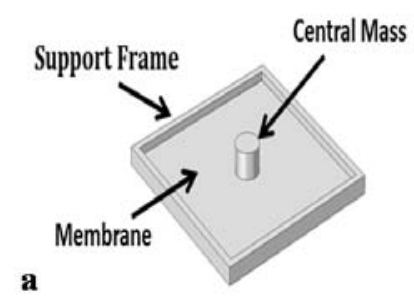

b

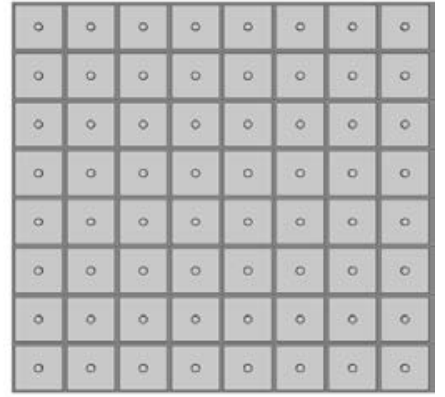

Figure 1. Membrane-type acoustic metamaterial model (a)Metamaterial element (b)Metamaterial

For studying the particular property of the membrane-type acoustic metamaterial, we simulated the transmission loss of the membrane-type acoustic metamaterial element by FEA(COMSOL Multiphysics was adopted). In this element, the center mass is chosen as lead with high mass density, and the weight of the center mass is $3.5 \mathrm{~g}$. The square membrane is chosen as high density 
polyethylene(HDPE) with the side length of $50 \mathrm{~mm}$ and the thickness of $0.16 \mathrm{~mm}$. The mass density, Young's modulus, the Poisson's ratio and prestress for the HDPE membrane are $1200 \mathrm{~kg} / \mathrm{m}^{3}$, $3.6 \times 10^{8} \mathrm{~Pa}, 0.32$ and $1 \mathrm{MPa}$,respectively. The mass density, Young's modulus, the Poisson's ratio for the rigid support frame are $2700 \mathrm{~kg} / \mathrm{m}^{3}, 7.1 \times 10^{10} \mathrm{~Pa}$, and 0.346 , respectively, with the edge width of $2 \mathrm{~mm}$ and the height of $4 \mathrm{~mm}$.

In the coupled acoustic-structural analysis, we established a membrane-type acoustic metamaterial model as shown in Fig.2(a). The sound source with plane wave incidences on left side of the model, and the right side is set as non-reflective boundary. The acoustic metamaterial is placed in the middle of the air cavity. After meshing with total model as shown in Fig.2(b), the element number of the membrane, central mass and support frame are 2296, 480, and 2340, respectively.
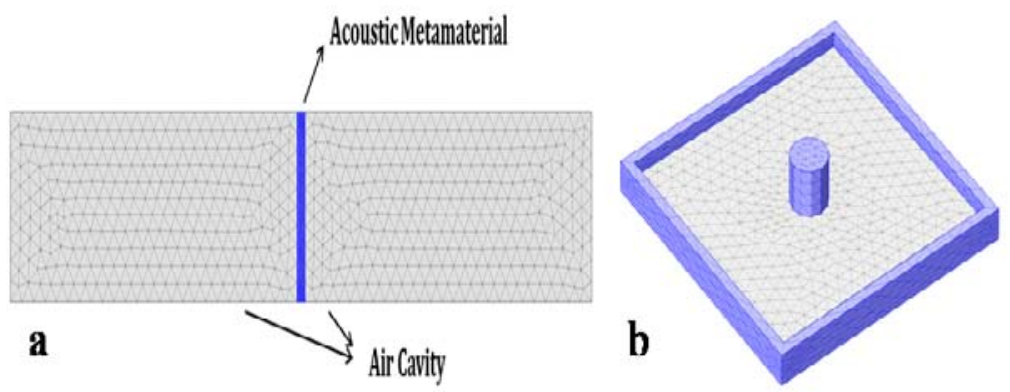

Figure 2. Finite Element Model of Membrane-type acoustic metamaterial(a) Acoustic-structure model(b)Mesh element distribution

Fig.3 presents the TL curves for the membrane-type acoustic metamaterial element and for the mass law calculation. For membrane-type acoustic metamaterial, the TL exceeds $20 \mathrm{~dB}$ in a frequency range from $42 \mathrm{~Hz}$ to $64 \mathrm{~Hz}$ as shown in Fig. 3 ; the TL arrives the maximum value of $41.19 \mathrm{~dB}$ when the sound frequency is $52 \mathrm{~Hz}$, but it is only $1.86 \mathrm{~dB}$ predicted by the mass law. We can see that the difference is an order of magnitude. When the sound frequency is $14 \mathrm{~Hz}$ and $16 \mathrm{~Hz}$, the TL is small, closely to 0dB. Fig.4 shows that the TL property of membrane-type acoustic metamaterial is dependent on its vibrationfeatures. The vibration direction of the central mass and the membrane is identical when the TL tends tovalley; while the vibration direction of the central mass and the membrane is opposite when the TL arrives the peak.

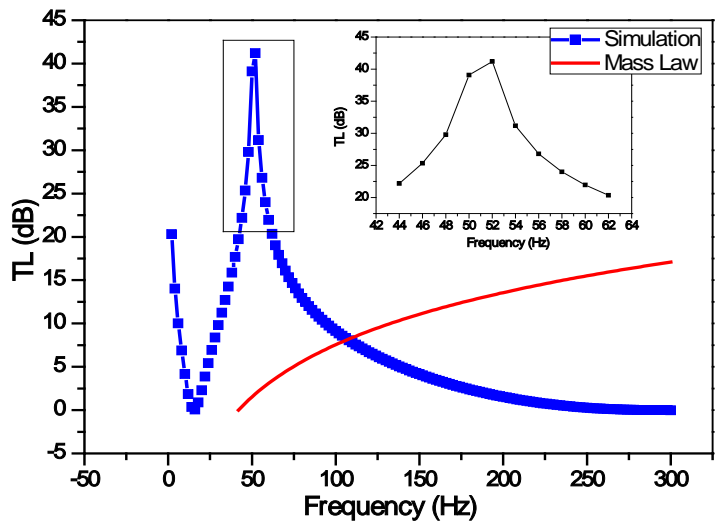

Figure 3. TL of membrane-type acoustic metamaterial and mass law prediction 

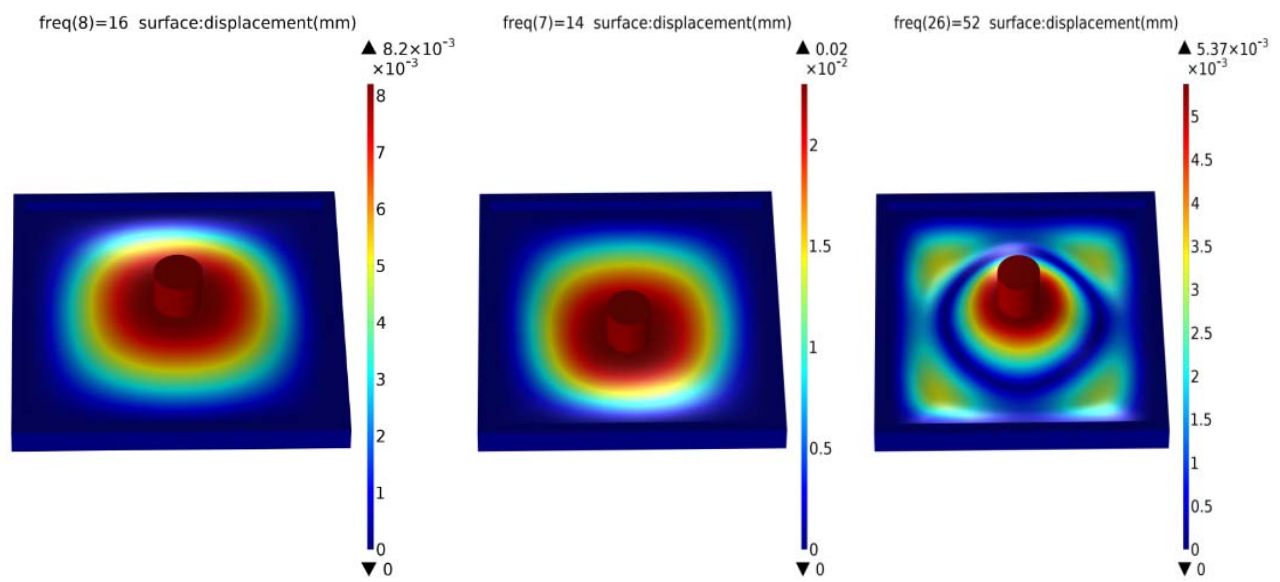

Figure 4. Coupled acoustic-structural analysis at different frequencies

Above calculated results indicate that the TL of membrane-type acoustic metamaterial is closely related to the vibration features, and the central mass plays a major role in influencing the vibration property. In the following, we will analyze the influences of center mass and the distribution type in details.

\section{Influence of mass on sound insulation characteristics}

The number, distribution type and mass magnitude of surrounding mass bricks on the membrane are sure to influence the sound insulation. This section firstly presents the role of centrally single mass, and then presents the sound insulation effect when the membrane-type acoustic metamaterial element is " $1+n$ Type"(" 1 " is the bigger mass located in the center of the elastic membrane, " $n$ " is the small masses distributing around the central mass here).

\subsection{TL of centrally single mass variation}

In order to understand the role of the central mass, we changed the weight of central mass, they were $1.75 \mathrm{~g}, 3.5 \mathrm{~g}$ and $7 \mathrm{~g}$, respectively, Fig. 5 shows TL curves at different situations. With the increase of mass magnitude, the peak of TL curve moves to low frequencies. This result indicates that it is beneficial for membrane-type acoustic metamaterial element absorbing low frequency noise when the central mass brick is heavy.

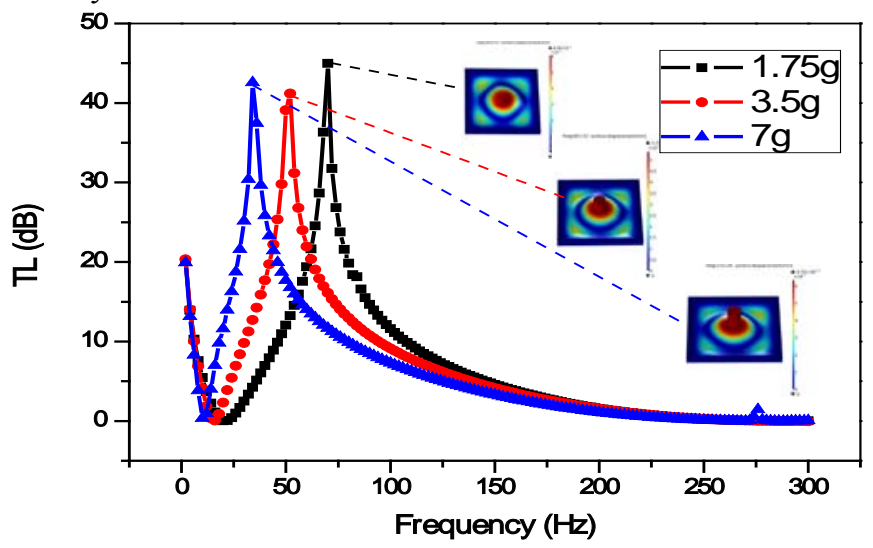

Figure 5. TL with central mass magnitude variation 


\subsection{TL effect of " $1+2$ Type"}

To further investigete the role of the number, distribution type and mass magnitude of surrounding mass bricks on the membrane, the following work designed a kind of membrane-type acoustic metamaterial called with " $1+2$ Type" as shown in Fig.6. In Fig.6(a), one mass(mass magnitude is M) is fixed on the center of membrane, and two small masses(mass magnitude is $\mathrm{m}$ ) are located at one diagonal line of the membrane. The distance from small mass to the membrane center is L. In Fig. (b), two small masses are located at one side of the membrane.
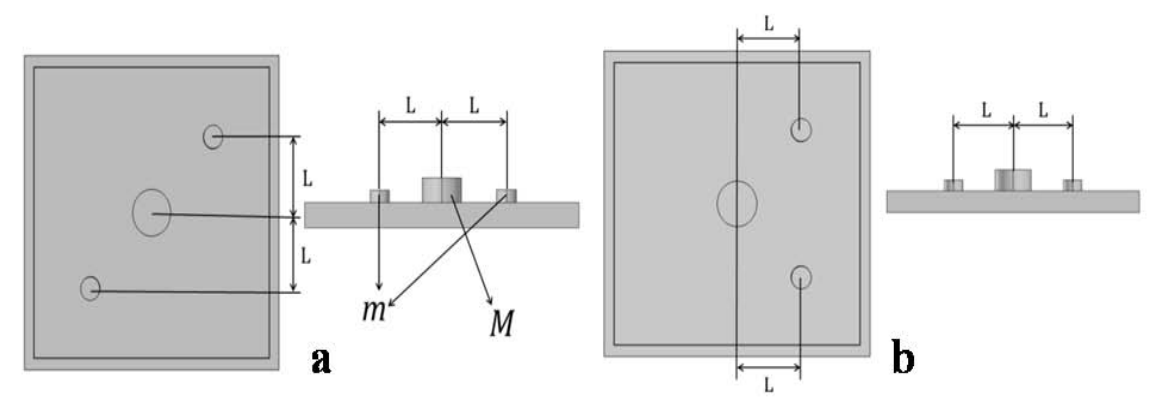

Figure 6. Acoustic metamaterial element of " $1+2$ Type" distribution(a)Diagonal line " $1+2$ Type" (b)Same side "1+2 Type"

Firstly, we analyze the sound insulation property of acoustic metamaterial element in Fig. $6(\mathrm{a})(\mathrm{L}=5 \mathrm{~mm}, 7 \mathrm{~mm}, 9 \mathrm{~mm}, 11 \mathrm{~mm}, 13 \mathrm{~mm}$, respectively, $\mathrm{M}=3.75 \mathrm{~g}, \mathrm{~m}=0.58 \mathrm{~g})$. The result presents in Fig.7. The TL curve of " $1+2$ Type" metamaterial element appears two peaks or three peaks. When $\mathrm{L}>9 \mathrm{~mm}$, the TL curve owns two peaks, and the change of L lightly influences the TL curve. When $\mathrm{L}<9 \mathrm{~mm}$, the TL curve presents three peaks, but the sound insulation frequency range of later peaks is extremely narrow.

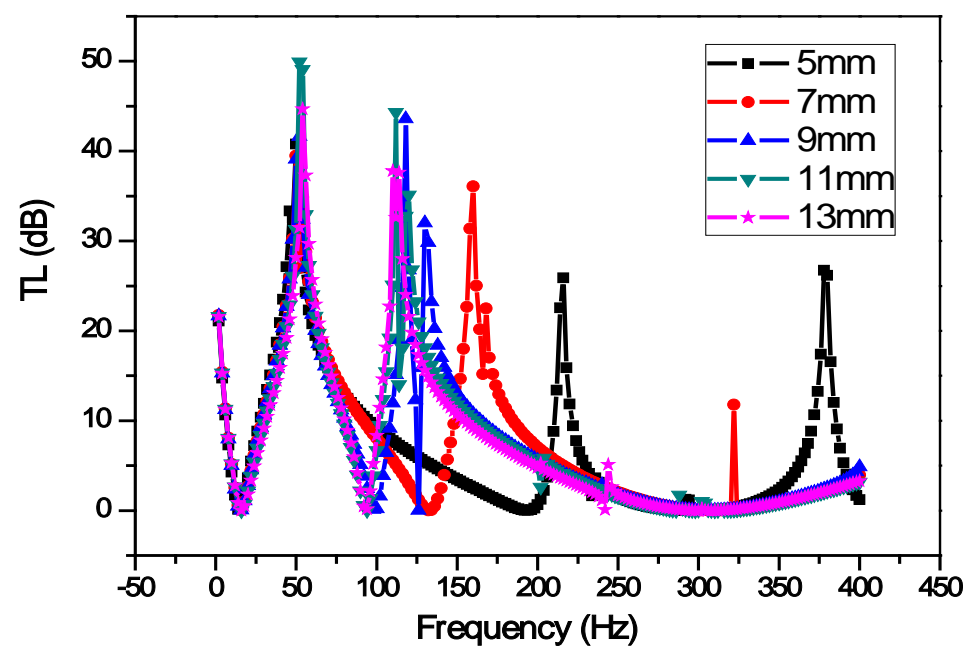

Figure 7. TL of " $1+2$ Diagonal Type" acoustic metamaterial element with $\mathrm{L}$ variation( $\mathrm{M}=3.75 \mathrm{~g}, \mathrm{~m}=0.58 \mathrm{~g})$

In order to study the role of mass magnitude of surrounding masses, we simulated the sound reduction of acoustic metamaterial element in Fig.6(a) ( $\mathrm{L}=13 \mathrm{~mm}, \mathrm{M}=3.75 \mathrm{~g}, \mathrm{~m}=0.15 \mathrm{~g}, 0.29 \mathrm{~g}, 0.58 \mathrm{~g}$, respectively). Fig. 8 presents that the first peak of the TL curve remains unchanged though the mass magnitude of small masses varies. However, the second peak of the TL moves to high frequencies, and the sound insulation range slowly narrows with the decrease of the mass magnitude of small masses. 


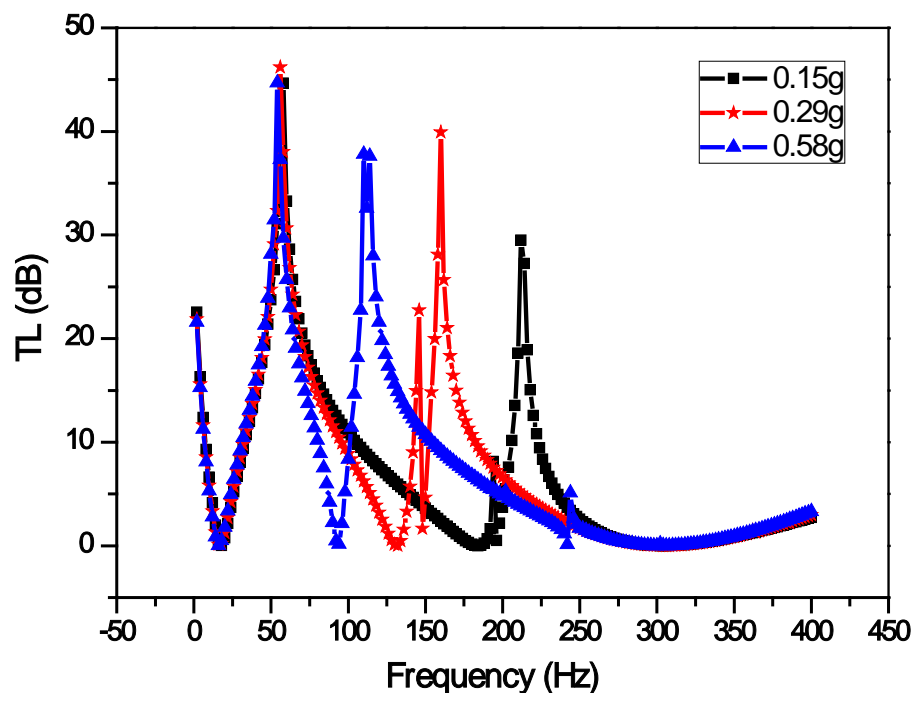

Figure 8. TL of " $1+2$ Diagonal Type" acoustic metamaterial element with $\mathrm{m}$ variation( $\mathrm{L}=13 \mathrm{~mm}, \mathrm{M}=3.75 \mathrm{~g})$

The distribution type of Diagonal Line "1+2 Type" is shown in Fig.6(a) and that of the Same Side "1+2 Type" is as shown in Fig.6(b). In order to understand the role of the distribution type, other variables remain unchanged $(\mathrm{M}=3.75 \mathrm{~g}, \mathrm{~m}=0.58 \mathrm{~g}, \mathrm{~L}=13 \mathrm{~mm})$. Fig. 9 shows that two peaks of the $\mathrm{TL}$ curve are almost identical at frequencies $(2 \mathrm{~Hz}-200 \mathrm{~Hz})$ though different acoustic metamaterial elements. The frequency at the first peak is $56 \mathrm{~Hz}$ and $54 \mathrm{~Hz}$, and the frequency at the second peak is $128 \mathrm{~Hz}$ and $114 \mathrm{~Hz}$, respectively. The TL curve has no marked difference if considering data error. This result indicates that the distribution type of small masses has no influence on the TL if the number of small masses is identical.

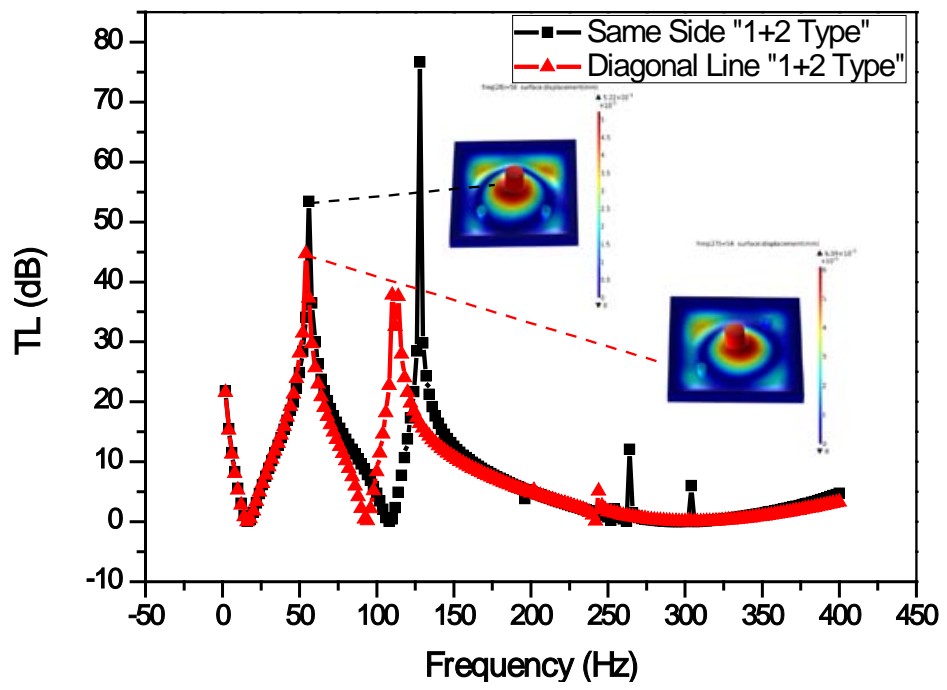

Figure 9. TL of Diagonal Line " $1+2$ Type" and Same Side " $1+2$ Type" $(\mathrm{L}=13 \mathrm{~mm}, \mathrm{M}=3.75 \mathrm{~g}, \mathrm{~m}=0.58 \mathrm{~g})$

\subsection{TL effect of triangular form " $1+3$ Type" and quadrilateral form "1+4 Type"}

Following work contrasts Triangular Form " $1+3$ Type" as shown in Fig. 10(a) with Quadrilateral Form "1+4 Type" as shown in Fig. 10(b). We acquired different sound insulation effects of the membrane-type acoustic metamaterial by changing the number of small masses. 
Fig.11 shows that " $1+2$ Type" appears two peaks, " $1+3$ Type" appears three peaks and " $1+4$ Type" appears four peaks. The peak number of the TL increases with the increase of small masses number. We also notice that the distance from the first peak to the second peak increases when $n$ increases. However, the first peak of TL remains basically unchanged though the number and the distribution type of small masses change greatly.
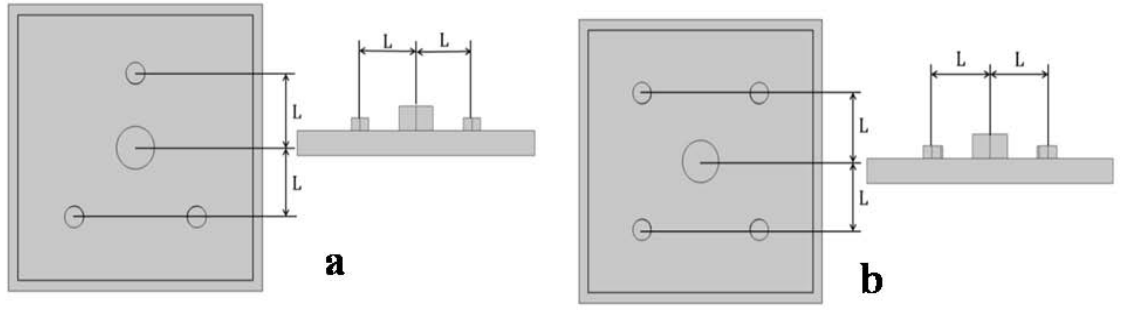

Figure 10. Acoustic metamaterial element (a)Triangular Form "1+3 Type" (b)Quadrilateral Form " $1+4$ Type"

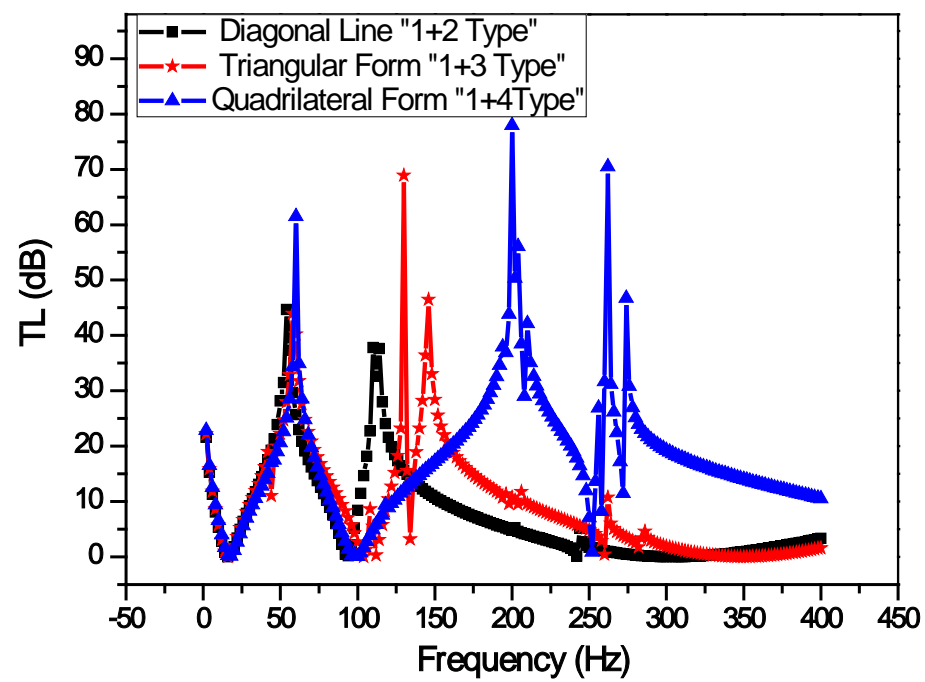

Figure 11. TL of membrane-type acoustic metamaterial $(\mathrm{n}=2,3,4)(\mathrm{M}=3.75 \mathrm{~g}, \mathrm{~m}=0.58 \mathrm{~g}, \mathrm{~L}=13 \mathrm{~mm})$

\section{Conclusions}

This work studies the sound insulation property of the membrane-type acoustic metamaterial.This membrane-type acoustic metamaterial can reduce the low frequency sound attenuation range to $20 \mathrm{~Hz}-$ $100 \mathrm{~Hz}$ and decrease the best sound insulation frequency greatly. We acquire the TL curve and vibration features by numerical simulation, the TL is the highest when the vibration direction of central mass and the membrane is opposite.

Small masses around central mass have an impact on TL curve, and we study the role of the number, distribution type and mass magnitude of small masses in details. The results indicate that the peak number of TL increase when increasing the number of small masses. The distribution type of small masses has light influence on TL when the number of small masses is identical. When the number and distribution type of small masses are the same, the mass magnitude of small masses changes the frequency in the second peak of the TL, but the first peak has no change. We only discuss the sound insulation property of the membrane-type acoustic metamaterial with finite masses, and more masses and distribution types need further study. 


\section{Acknowledgment}

This work was supported by the Fundamental Research Funds for the Central Universities (Grant No. HIT.MKSTISP. 201609).

\section{References}

1. D. Takahashi, K. Sakagami, and M. Morimoto, J. Acoust. Soc. Am. 99, 3003 (1996)

2. Z. Yang, J. Mei, M. Yang, N. H. Chan, and P. Sheng, Phys. Rev. Lett. 101, 204301 (2008)

3. Z.Yang, H. M. Dai, N. H. Chan, G. C. Ma, and P. Sheng, Appl. Phys. lett. 96, 041906 (2010)

4. C. J. Naify, C. M.Chang, G. McKnight, and S. Nutt, J. Appl. Phys. 108, 114905 (2010)

5. C. J. Naify, C. M.Chang, G. McKnight, F. Schenlen, and S. Nutt, J. Appl. Phys. 109,104902 (2011)

6. C. J. Naify, C. M.Chang, G. McKnight, and S. Nutt, J. Appl. Phys. 110, 124903 (2011)

7. C. J. Naify, C. M.Chang, G. McKnight, and S. Nutt, J. Acoust. Soc. Am. 132,2784 (2012)

8. Y. Y. Chen, G. L. Huang, X. M. Zhou, G. K. Hu, and C. T. Sun, J. Acoust. Soc. Am. 136, 969 (2014)

9. H. Y. Tian, X. Z. Wang, and Y. H. Zhou, Appl. Phys. A. 114, 985 (2014)

10. G. C. Ma, M. Yang, Z. Y. Yang,and P. Sheng, Appl. Phys. Lett. 103,011903 (2013)

11. M. Yang, Y. Li, C. Meng, C. X. Fu, J. Mei, Z. Y. Yang, and P. Sheng, C. R. Mecanique, 343,635(2015)

12. J. Mei, G. C. Ma, M. Yang, Z. Y. Yang, W. J. Wen, and P. Sheng, Nat. Commun.3,756 (2012)

13. Y. G. Zhang, J. H. Wen, Y. Xiao, X. S. Wen, and J. W. Wang, Phys. Lett. A. 376, 1489 (2012)

14. J. S. Bolton, N. M. Shiau, and Y. J. Kang, J. Sound Vib. 191, 317 (1996) 